\title{
Information Filtering via Biased Random Walk on Coupled Social Network
}

\author{
Da-Cheng Nie, ${ }^{1}$ Zi-Ke Zhang, ${ }^{2,3}$ Qiang Dong, ${ }^{1}$ Chongjing Sun, ${ }^{1}$ and Yan Fu ${ }^{1}$ \\ ${ }^{1}$ Web Sciences Center, School of Computer Science \& Engineering, University of Electronic Science and Technology of China, \\ Chengdu 610054, China \\ ${ }^{2}$ Institute of Information Economy, Hangzhou Normal University, Hangzhou 311121, China \\ ${ }^{3}$ Alibaba Research Center for Complexity Sciences, Hangzhou Normal University, Hangzhou 311121, China
}

Correspondence should be addressed to Da-Cheng Nie; niedacheng@gmail.com

Received 27 March 2014; Revised 30 June 2014; Accepted 8 July 2014; Published 22 July 2014

Academic Editor: Yolanda Blanco Fernandez

Copyright (C) 2014 Da-Cheng Nie et al. This is an open access article distributed under the Creative Commons Attribution License, which permits unrestricted use, distribution, and reproduction in any medium, provided the original work is properly cited.

\begin{abstract}
The recommender systems have advanced a great deal in the past two decades. However, most researchers focus their attentions on mining the similarities among users or objects in recommender systems and overlook the social influence which plays an important role in users' purchase process. In this paper, we design a biased random walk algorithm on coupled social networks which gives recommendation results based on both social interests and users' preference. Numerical analyses on two real data sets, Epinions and Friendfeed, demonstrate the improvement of recommendation performance by taking social interests into account, and experimental results show that our algorithm can alleviate the user cold-start problem more effectively compared with the mass diffusion and user-based collaborative filtering methods.
\end{abstract}

\section{Introduction}

In the past two decades, the Web 2.0 and its applications have greatly accelerated the development of the Internet. They bring our lives much convenience as well as overwhelm us with too many resources in the information ocean. One typical scenario is online shopping in our daily life. When we are confronted with millions of books on http://www.Amazon.com or billions of different kinds of commodities on http://www.Taobao.com, indeed, it is very difficult to choose the relevant ones from countless candidates. This is the so-called Information Overload problem [1]. Therefore, an automatic way that can help us make the right decision under the Information Overload is a significant issue for both academic and industrial communities.

Search engines provide a way to help users find the useful information, which alleviates this dilemma partially: a user inputs the keywords and then the search engine returns the results accordingly. However, if different users input the same keywords, the search engine will return the same results. Besides, when users resort to a search engine, they must know how to clearly describe what they want by the keywords. But in most situations, users do not know what they really want or it is hard for them to find appropriate keywords. In this case, the recommender systems [2] have been designed to solve this problem.

Recently, social networks (SN) $[3,4]$ have become a powerful tool to characterize social relationship in online social services, emerging with various Web 2.0 applications [5] in evolutionary games [6, 7], community detection [8], medical science [9], and so forth. By taking advantage of social relationship in recommender systems, many traditional challenges can be partially solved, such as the cold-start problem [10] and data sparsity problem [11]. However, most researches are focused on mining the similarities among users or objects in recommender systems, and the social influence is seldom taken into account.

Coupled networks (CN), also known as interdependent networks [12], are usually composed of two layers of networks [12, 13], such as electricity/internet networks [14] and airport/railway networks [15]. Being similar with interdependent networks, a coupled social network (CSN) also contains the coupling nodes (users), which form a leader-follower relationship in the layer of social network and collecting 
relationship in the layer of information network. Figure 1 gives an illustration of a simple CSN with five users and five objects, where circles denote users and squares represent objects; the social network (upper layer) consists of five users and the information network (lower layer) consists of five objects and five users, where the users are the same as those in the social network. It can be seen that $O_{5}$ will not be recommended to $U_{4}$ in the user-object network because only $U_{5}$ collects object $O_{5}$ and the value of similarity between $U_{4}$ and $U_{5}$ is zero. However, $U_{4}$ follows $U_{5}$ in the social network, which indicates that $U_{4}$ may have similar interests with $U_{5}$ to some extent; thus, we can accordingly recommend $O_{5}$ to $U_{4}$ via social network. Therefore, by making use of the social relationship between users, the user cold-start problem can be partially solved. When a new user comes to the system, we can recommend him/her some objects through the social network.

Moreover, most researchers focus their attention on mining the similarities among users or objects in recommender systems, and many researchers use the social interest to filter the recommendations, but we use the social interest to supplement the recommendations instead of filtering them. To our knowledge, the random walk algorithm on coupled social network remains yet to be investigated in recommender systems.

The contributions of this paper can be summarized as follows. (1) We use the social interest to supplement the recommendations instead of filtering them and we obtain more accurate recommendations. (2) We first propose a biased random walk recommendation algorithm on coupled social network, which considers the social interests as well as users' preference in the recommender systems. This method can improve the performance of recommendations. (3) Compared with the mass diffusion (MD) $[16,17]$ and user-based CF (UCF) $[18,19]$ methods, the proposed algorithm can alleviate the user cold-start problem more effectively.

This paper is organized as follows. We introduce the related works in Section 2. In Section 3, we propose a biased random walk algorithm on coupled social network. In Section 4 we describe the data sets and metrics used in this paper. We evaluate the performance of the proposed method in Section 5. Finally, we summarize this paper in Section 6.

\section{Related Works}

Collaborative filtering (CF) [18-25] is the most frequently used technology in recommender systems, which uses the collection history of users for mining the potential objects of interest to the target user. However, the CF algorithm only takes the similar users or objects into account and will lead to the same recommendation results to diverse users; namely, it is not conducive to the personalized recommendation. Meanwhile, the CF algorithm cannot deal with the cold-start problem [10]; that is, when a new user or object is added to the system, it is difficult to obtain recommendation or to be recommended because of lack of enough information. To alleviate this problem, many methods have been proposed, such as content-based [26], trust-aware [27, 28], socialimpact [29], and tag-aware [30] methods.

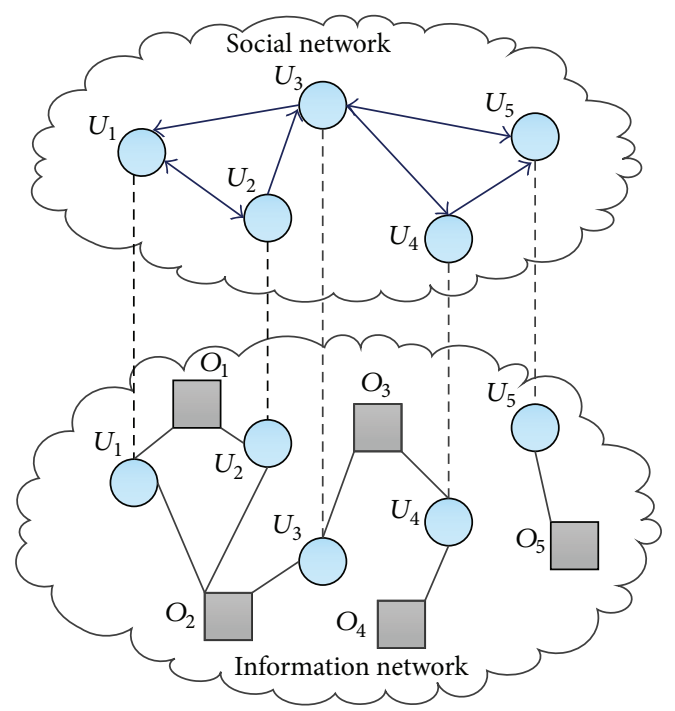

FIGURE 1: Illustration of a coupled social network with five users and five objects, where circles denoteusers and squares represent objects (color online). The social network (upper layer) consists of five users; the information network (lower layer) consists of five objects and five users, while user nodes are the same in the social network.

Random walk [31] is a mathematical formalization of a path that consists of a succession of random edges, which is successfully used in recommender systems based on bipartite network [16, 32], namely, mass diffusion (MD for short) method [16]. Accordingly, many methods based on mass diffusion were proposed [17, 33]. Furthermore, random walk was successfully used in many fields, such as social network [34] and Top- $k$ search [35]. However, there is a lack of study of random walk on coupled social network in recommender systems.

Massa and Avesani [36] proposed a social propagation method that is based on users' distance from a fixed propagation horizon, which increased the coverage of recommender systems. Esslimani et al. [37] proposed a feedback effect between similarity and social influence in online communities. By utilizing the social relations, we can obtain the strength of social relationship between users, and we can use this social relationship to generate more accurate recommendation results. Meanwhile, the literature [36, 38] demonstrated that recommendation performance can be improved by taking into consideration the effect of social network, and the methods are both filtering the useless information by social relationship.

Lai et al. [39] proposed a hybrid personal trust model which adaptively combines the rating-based trust model and explicit trust metric to resolve the drawback caused by insufficient past rating records. Community-based recommender systems have attracted much research attention; the authors [40] proposed a novel community-based framework that employs PLSA-based model incorporating social activeness and dynamic interest to discover communities. Wei et al. [41] proposed a multicollaborative filtering trust network algorithm, an improved version of CF algorithm designed to work 
on Web 2.0 platform, which can improve the prediction accuracy compared with the original CF algorithm. We believe that if the social relationship can be used to supplement the user-object network like the aforementioned example of Figure 1, we will get more accurate recommendations and alleviate the user cold-start problem. Motivated by this, we proposed a biased random walk (diffusion-based) method on coupled social network to generate recommendations. Therefore, new users can obtain recommendations as long as they are connected to others in social networks.

\section{Method}

In this section, we introduce the approach of diffusion on coupled social networks. Generally, a recommender system consists of two sets, $U=\left\{U_{1}, U_{2}, \ldots, U_{m}\right\}$ and $O=$ $\left\{O_{1}, O_{2}, \ldots, O_{n}\right\}$ representing the $m$ users and $n$ objects, respectively. Denote $A_{m \times n}$ by the adjacent matrix of the userobject bipartite network, of which each element $a_{i \alpha}=1$, if user $U_{i}$ has collected object $O_{\alpha}$, and $a_{i \alpha}=0$ otherwise. Analogously, denote $B_{m \times m}$ by the nonsymmetric adjacent matrix of user-user directed social network, of which each element $b_{i j}=1$, if the user $U_{i}$ has linked to user $U_{j}$, and $b_{i j}=0$ otherwise.

Random Walk on Social Network. Let $P^{\prime}$ be the $m \times m$ transition probability matrix of a directed social network. The probability that a random walker at user $U_{i}$ goes to user $U_{j}$ on social network can be described as

$$
p_{i j}^{\prime}= \begin{cases}\frac{b_{i j}}{k_{i}^{\text {out }}}, & \text { if } k_{i}^{\text {out }} \neq 0 \\ 0, & \text { otherwise }\end{cases}
$$

where $k_{i}^{\text {out }}$ is the out-degree in social network, that is, the number of leaders of user $U_{i}$. Denote $s_{i}^{\prime}(t)$ by the probability from other users to user $U_{i}$ at time $t$. Therefore, we have

$$
s_{i}^{\prime}(t+1)= \begin{cases}\sum_{j=1}^{m} \frac{b_{i j}}{k_{i}^{\text {out }}} s_{j}^{\prime}(t), & \text { if } k_{i}^{\text {out }} \neq 0 \\ 0, & \text { otherwise. }\end{cases}
$$

The initial probability for target user $U_{i}$ is given by $s_{i}^{\prime}(0)=1$, and $s_{j}^{\prime}(0)=0$ for all of the other user $U_{j}$. Thus, we can obtain the probability that a random walker goes from the target user to all other users at time $t$.

Random Walk on Bipartite Network. Let $P^{\prime \prime}$ be the $m \times$ $n$ transition probability matrix of a bipartite network. The probability that a random walker at user $U_{i}$ goes to object $O_{\alpha}$ on bipartite network can be described as

$$
p_{i \alpha}^{\prime \prime}= \begin{cases}\frac{a_{i \alpha}}{k_{i}}, & \text { if } k_{i} \neq 0 \\ 0, & \text { otherwise }\end{cases}
$$

where $k_{i}$ denotes the number of collected objects of user $U_{i}$, and the probability that a random walker at object $O_{\alpha}$ goes to user $U_{j}$ on bipartite network can be described as

$$
p_{\alpha j}^{\prime \prime}= \begin{cases}\frac{a_{j \alpha}}{k_{\alpha}}, & \text { if } k_{\alpha} \neq 0 \\ 0, & \text { otherwise }\end{cases}
$$

where $k_{\alpha}$ denotes the number of users who have collected object $O_{\alpha}$ on bipartite network. Denote $s_{i}^{\prime \prime}(t)$ and $s_{\alpha}^{\prime \prime}(t)$ by the probability of user $U_{i}$ and object $O_{\alpha}$ on bipartite network at time $t$, respectively. Therefore, we have

$$
\begin{aligned}
& s_{i}^{\prime \prime}(t+1)= \begin{cases}\sum_{\alpha=1}^{n} \frac{a_{i \alpha}}{k_{i}} s_{\alpha}^{\prime \prime}(t), & \text { if } k_{i} \neq 0 \\
0, & \text { otherwise, }\end{cases} \\
& s_{\alpha}^{\prime \prime}(t+1)= \begin{cases}\sum_{j=1}^{m} \frac{a_{j \alpha}}{k_{\alpha}} s_{j}^{\prime \prime}(t), & \text { if } k_{\alpha} \neq 0 \\
0, & \text { otherwise. }\end{cases}
\end{aligned}
$$

Similar to random walk on social network, the initial probability for target user $U_{i}$ is given by $s_{i}^{\prime \prime}(0)=1$. But the difference is the fact that there are two different nodes on bipartite network and the initial probability $s_{j}^{\prime \prime}(0)=0$ and $s_{\alpha}^{\prime \prime}(0)=0$ for all the other user $U_{j}$ and object $\alpha$. In the odd time step and $t \geq 3$, the probability of $s_{\alpha}^{\prime \prime}(t)$ means the probability of target user $U_{i}$ selecting uncollected object $O_{\alpha}$. Therefore, we can obtain the recommendation list according to this probability for target user.

Biased Random Walk on Coupled Social Network. Let $P$ be the $M \times M$ transition probability matrix of a coupled social network, where $M=m+n$. In order to solve the user coldstart problem, suppose that a random walker at user $U_{i}$ goes to their neighbors (leaders) on directed social network with probability $\lambda \in(0,1)$, and to their neighbors on bipartite network with probability $1-\lambda$. What's more, a random walker at object $O_{\alpha}$ goes to all users who collect object $O_{\alpha}$ with equal probability. Thus, the target user finds the potential objects not only through other users with similar collecting interest on bipartite network, but also through their friends (leaders) on directed social network. Denote $s_{i}(t)$ and $s_{\alpha}(t)$ by the probability of walker user $U_{i}$ and object $O_{\alpha}$ on coupled social network at time $t$, respectively. Therefore, we have

$$
s_{i}(t+1)= \begin{cases}\lambda \cdot \sum_{j=1}^{m} \frac{b_{i j}}{k_{i}^{\text {out }}} s_{j}(t)+(1-\lambda) & \\ \cdot \sum_{\alpha=1}^{n} \frac{a_{i \alpha}}{k_{i}} s_{\alpha}(t), & \text { if } k_{i} \neq 0, k_{i}^{\text {out }} \neq 0 \\ \sum_{j=1}^{m} \frac{b_{i j}}{k_{i}^{\text {out }}} s_{j}(t), & \text { if } k_{i}=0, k_{i}^{\text {out }} \neq 0 \\ \sum_{\alpha=1}^{n} \frac{a_{i \alpha}}{k_{i}} s_{\alpha}(t), & \text { if } k_{i} \neq 0, k_{i}^{\text {out }}=0 \\ 0, & \text { otherwise, }\end{cases}
$$




$$
s_{\alpha}(t+1)= \begin{cases}\sum_{j=1}^{m} \frac{a_{j \alpha}}{k_{\alpha}} s_{j}(t), & \text { if } k_{\alpha} \neq 0 \\ 0, & \text { otherwise. }\end{cases}
$$

That is to say, initially, we assign the target user one unit of resource. Then $\lambda(0 \leq \lambda \leq 1)$ proportion of the resource is evenly distributed to the user's social neighbors through the directed links (social network), and $1-\lambda$ proportion is distributed to collected objects through the undirected links (bipartite network). In (6), when $k_{i}^{\text {out }}=0$ then $\lambda=0$; it means that user $U_{i}$ has no outlinks in social network; therefore, he/she will distribute all of his/her resources to bipartite network. Similarly, when $k_{i}=0$, then $\lambda=1$; user $U_{i}$ will distribute all of his/her resources to social network.
The initial score for target user $U_{i}$ is given by $s_{i}(0)=1$, $s_{j}(0)=0$, and $s_{\alpha}(0)=0$ for all the other user $U_{j}$ and object $\alpha$. Thus, we can obtain the recommendations by ranking the score $s_{\alpha}$ of all objects at time $t$ for target user. At time $t=2$, the recommendations are obtained only from social network, that is, his/her social leaders. At time $t=3$ and $\lambda=0$, the recommendations are obtained only from bipartite network and it is the pure MD algorithm.

Thus, the probability that a random walker arrives at the object at time $t$ is recognized as the possibility that the target user purchases this object. We call this algorithm biased random walk (BRW). For the example in Figure 1, the transition probability matrix $P$ for coupled social network is given in the following equation:

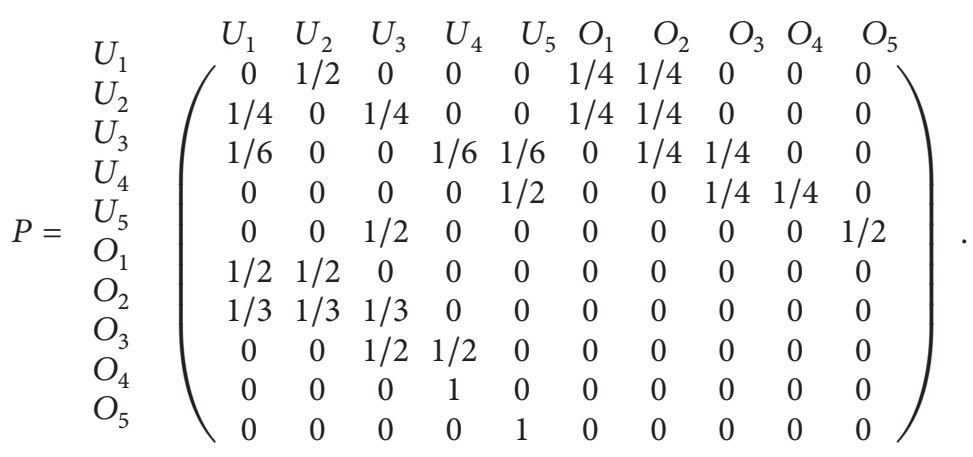

Consider $\lambda=0.5$ and $t=2$; then $P\left(U_{3}, O_{5}\right)=0.0833$, which means users $U_{3}$ and $O_{5}$ are reachable within 2 steps with 0.0833 probability through the coupled social network. On the other hand, without social network, the random walk distance on the original bipartite network $P^{\prime \prime}\left(U_{3}, O_{5}\right)=0$ for an arbitrary time $t$ because $U_{3}$ and $O_{5}$ are not reachable from each other in bipartite network.

\section{Data and Metrics}

4.1. Data Sets. To evaluate our algorithm's performance, two real data sets are analyzed in the experiments. The data sets are from http://www.epinions.com and http:// www.friendfeed.com, both of which provided user-objects collecting information and user-user social relationship. The Epinions data set was collected by Paolo Massa in a 5-week crawl (November/December 2003) from the http://www.epinions.com website [36] and the Friendfeed data set was collected by Fabio Celli et al. from http:// www.friendfeed.com (September 6, 2009 to September 19, 2009) [42]. We extract a smaller data set by randomly sampling the whole records of user activities in both Epinions and Friendfeed data sets. 4,066 users, 7,649 objects, 154,122 collected links, and 217,071 social links in total were found in the Epinions data set. Friendfeed contains 4,148 users who collected 5,700 objects, 96,942 collected links, and 386,804 social links. Table 1 shows the basic statistics for two representative data sets. Denote $|U|,|O|$, and $N_{R}$ by the number of users, objects, and ratings, respectively. Sparsity $=N_{R} /(|U| \times|O|)$ denotes the data sparsity of user-objects network.

4.2. Metrics. To test our algorithm's performance, each information network is randomly divided into two parts: the training set consists of $90 \%$ entries and the remaining entries constitute the testing set. The training set is treated as known information used for generating recommendations, while the training set is regarded as unknown information used for testing the performance of the recommendation results. To evaluate the proposed algorithm, we employed five different metrics that characterize not only the accuracy of recommendations, but also the diversification, which are defined as follows.

(1) Precision [43]. Precision represents the probability that the selected objects appeared in the recommendation list which is shown as

$$
\operatorname{Precision~}_{i}=\frac{N_{r s}^{i}}{L},
$$

where Precision ${ }_{i}$ represents user $u_{i}$ 's precision, $N_{r s}^{i}$ denotes the number of recommended objects that appeared in the $U_{i}$ 's testing set, and $L$ represents the length of recommendation 
TABLE 1: Properties of the tested data sets.

\begin{tabular}{lccccc}
\hline Data sets & Users & Objects & Collecting links & Social links & Sparsity \\
\hline Epinions & 4,066 & 7,649 & 154,122 & 217,017 & $5 \times 10^{-3}$ \\
Friendfeed & 4,148 & 5,700 & 96,942 & 386,804 & $4.1 \times 10^{-3}$ \\
\hline
\end{tabular}

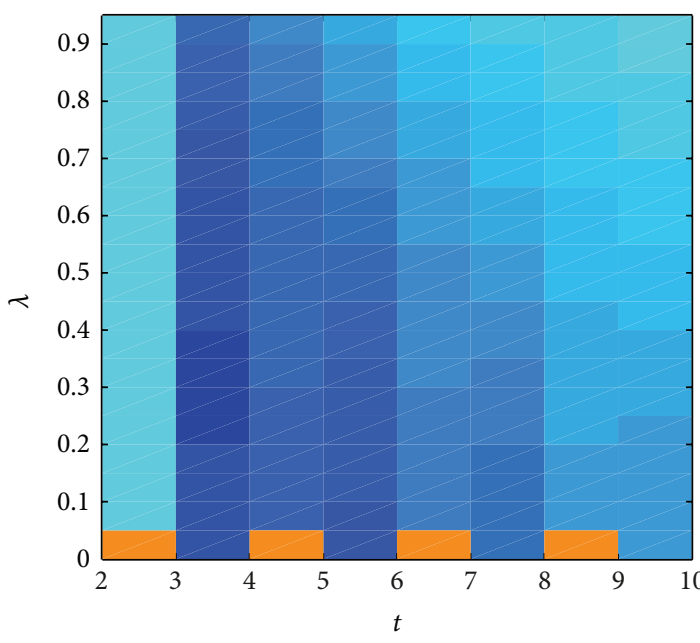

(a) Epinions



FIGURE 2: Ranking score values on Epinions and Friendfeed data sets (color online). list. By averaging over all users' precisions, we can obtain the whole recommender systems' precision as

$$
\text { Precision }=\frac{1}{m} \sum_{i=1}^{m} \text { Precision }_{i},
$$

where $m$ represents the number of users. Obviously, a higher precision means a higher recommendation accuracy.

(2) Recall [43]. Recall represents the probability that the recommended objects appeared in user's collected list shown as

$$
\operatorname{Recall}_{i}=\frac{N_{r s}^{i}}{N_{p}^{i}}
$$

where Recall ${ }_{i}$ represents user $u_{i}$ 's recall and $N_{p}^{i}$ is the number of objects collected by user $u_{i}$ in the testing set. Averaging over all individuals' recall, we can obtain the recall of the whole recommender system.

(3) F-Measure [43]. Generally speaking, for each user, recall is sensitive to $L$ and a larger $L$ generally gives a higher recall but a lower precision. The $F$-measure, that assigns equal weight for precision and recall, is defined as

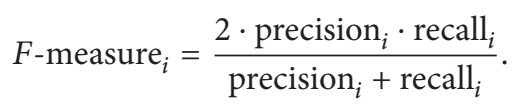

By averaging over all users' $F$-measure, we can also obtain the whole system's $F$-measure.

(4) $\mathrm{HD}$ [17]. HD is a metric to measure the diversity of users' recommendation lists. It uses the Hamming distance to measure the difference of recommendation lists between users $u_{i}$ and $u_{j}$, which is defined as

$$
\mathrm{HD}_{i j}(L)=1-\frac{Q_{i j}(L)}{L},
$$

where $Q_{i j}(L)$ is the number of commonly recommended objects shown in top- $L$ locations of users $u_{i}$ and $u_{j}$ 's recommendation list. Averaging over all pairs of users' $\mathrm{HD}_{i j}(L)$, we can obtain the HD of the recommender algorithm. Obviously, higher HD means higher diversity of users.

(5) Ranking Score ( $r$ ) [44]. Generally, the recommender system aims to generate a ranking list for the target user's uncollected objects through the prediction score. In the recommender systems, one of the most used metrics to evaluate the algorithm's performance is ranking score, which measures the users' satisfaction of the ranking list, and is defined as follows:

$$
r_{i \alpha}=\frac{L_{i \alpha}}{N_{i}}
$$

where $L_{i \alpha}$ is the position of uncollected object $\alpha$ in user $U_{i}$ 's ranking list and $N_{i}$ is the length of the user $U_{i}$ 's ranking list. By averaging all links' ranking score value we can obtain the whole system's ranking score value $r$. A small $r$ means the recommender system puts the user's favorite objects in a top place in the recommender list; hence, the smaller $r$ is, the better an algorithm's performance will be. 
TABLE 2: Algorithmic performance for Epinions data set with recommendation list $L=20$.

\begin{tabular}{lccccc}
\hline Method & $r$ & Precision & Recall & $F$-measure & HD \\
\hline MD & 0.172 & 0.036 & 0.099 & 0.046 & 0.041 \\
UCF & 0.186 & 0.033 & 0.090 & 0.56 \\
RW & $\mathbf{0 . 1 7 1}$ & $\mathbf{0 . 0 3 6}$ & $\mathbf{0 . 1}$ & $\mathbf{0 . 0 4 6}$ & 0.652 \\
\hline
\end{tabular}

TABLE 3: Algorithmic performance for Friendfeed data set with recommendation list $L=20$.

\begin{tabular}{lccccc}
\hline Method & $r$ & Precision & Recall & $F$-measure & HD \\
\hline MD & 0.116 & 0.03 & 0.140 & 0.041 & 0.9405 \\
UCF & 0.12 & 0.029 & 0.0902 & 0.0386 & 0.8772 \\
RW & $\mathbf{0 . 1 0 8}$ & $\mathbf{0 . 0 3}$ & $\mathbf{0 . 1 4 1}$ & $\mathbf{0 . 0 4 1}$ & 0.9250 \\
\hline
\end{tabular}

\section{Results}

Figure 2 shows the ranking score values on Epinions and Friendfeed data sets. From the figure we can see that the best performance is achieved at time $t=3$. At time $t=2$, the recommendations are obtained only from social network and when $\lambda=0$ it will generate random recommendation results since the ranking score value $r$ is much bigger than others. When $\lambda=0$ the resource will spread only on bipartite network; therefore objects get scores in odd time steps only, and user get scores in even time steps only. In addition, the ranking score will fluctuate up and down alternately with time $t$. That is because when $\lambda>0$ the recommendations are obtained from social interest in odd time step, and from both social interests and collecting preferences in even time step. With the increase of time $t$ in even and odd time step, respectively, the ranking score becomes worse due to the existence of the redundant correlations [45].

The best ranking score performance occurs at time $t=3$; that is, when we consider the social interest in the recommender systems, it will improve the performance of recommender systems. Figure 3 shows the experimental results of precision, recall, $F$-measure, HD with recommendation list $L=20$, and ranking score $r$ on Epinions and Friendfeed data sets at time $t=3 . \lambda=0$ gives the pure $\mathrm{MD}$ algorithm. It can be found that when the parameter $\lambda$ reaches the optimal value, the precision, recall, $f$-measure, and $r$ almost simultaneously reach the maximum value except that of HD. Tables 2 and 3 show the results of biased random walk (BRW) compared with the mass diffusion (MD) and user-based CF (UCF) on Epinions and Friendfeed data sets, respectively. We can see that BRW algorithm has a higher ranking-accuracy than other algorithms and almost similar accuracy-precision with MD but lower diversity-precision than MD algorithm. It is because the probability of reciprocity links $r_{L}=L^{\leftrightarrow} / L^{\prime}$ is large in the social network (Epinions data set is $45.47 \%$ and Friendfeed data set is $62.72 \%$ ), where $L^{\leftrightarrow}$ is the number of bidirectional links and $L^{\prime}$ is the number of all links in social network. Because it is easier for the random walker to go from one user to another user in social network, the recommendations obtained from social network will be similar among friends.
Generally speaking, the small degree users are the vast majority in the systems (Figure 4 shows the use degree distribution in the training set on Epinions and Friendfeed data sets. We find that there are $23.06 \%$ and $61.5 \%$ users with degrees smaller than 10 on Epinions and Friendfeed data sets, resp.). That is to say, increasing the small degree users' performance could result in performance improvement of the whole system. In Figure 5, we show the effect of user degrees that is in the training set versus ranking score. From the figure we can see that the MD and UCF almost have the same ability for small degree users and our method has better performance than MD and UCF algorithm. Meanwhile, it can be seen that our method considering the social interest into the recommender system has a better performance for both larger and smaller degree users. In other words, it can alleviate the user cold-start problem.

\section{Conclusion and Discussion}

In a real online recommender system, for new users or users with less collections, it is difficult to obtain recommendations because of lack of enough information. However, if they are active in the social network, the system can obtain the recommendations from their friends or social leaders. In this way, the social networks can help us to solve the user coldstart problem.

In this paper, we proposed a recommendation algorithm via biased random walk on a two-layer coupled network: user-object bipartite network and user-user social network. Experiment results on two real data sets indicate that social interest and user's preference can be combined together in a delicate way to improve the accuracy metric of recommendation systems. Compared with two other baseline algorithms, our algorithm achieves the best precision measure and has the best ability of accurately recommending objects to the small degree users, effectively alleviating the user cold-start problem.

This paper only provides a simple method to incorporate the social interest into the recommender systems by random walk on coupled social-information network, while a couple 

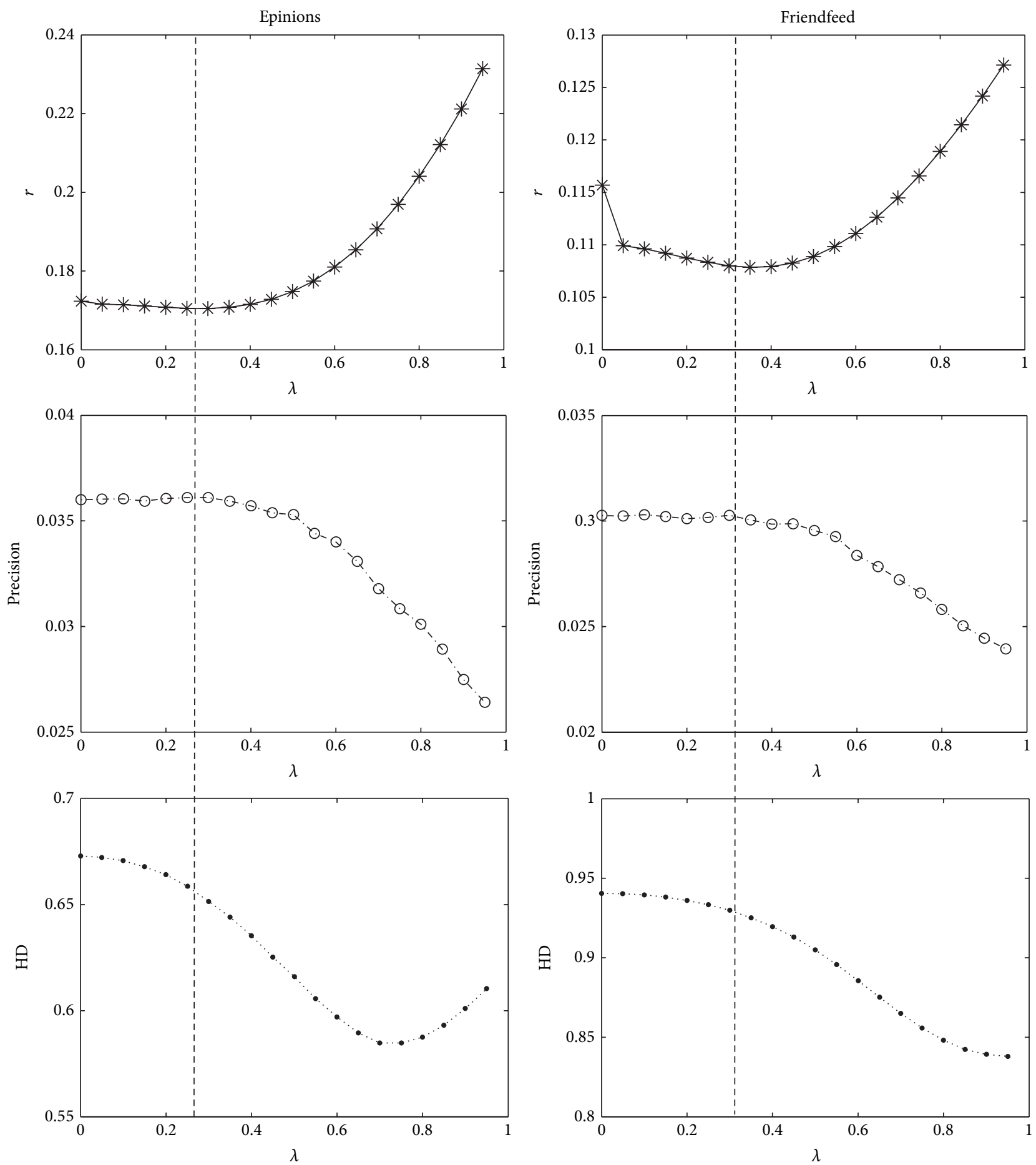

FIGURE 3: The precision and HD when recommendation list $L=20$ and $r$ in the Epinions and Friendfeed data sets. Each result is obtained by averaging over 10 independent runs, each of which corresponds on a random division of training set and testing set.

of issues remain open for future study. (i) The structure and evolution of coupled social networks are still unclear to us, but we believe they will be helpful for designing effective recommendation algorithms. (ii) The current algorithm assumes that a random walker goes to his friend on social network and his collected objects on bipartite network with the same probability; we conjecture that an appropriately adjusted weight assignment will further improve the algorithmic performance.

\section{Conflict of Interests}

The authors declare that there is no conflict of interests regarding the publication of this paper. 

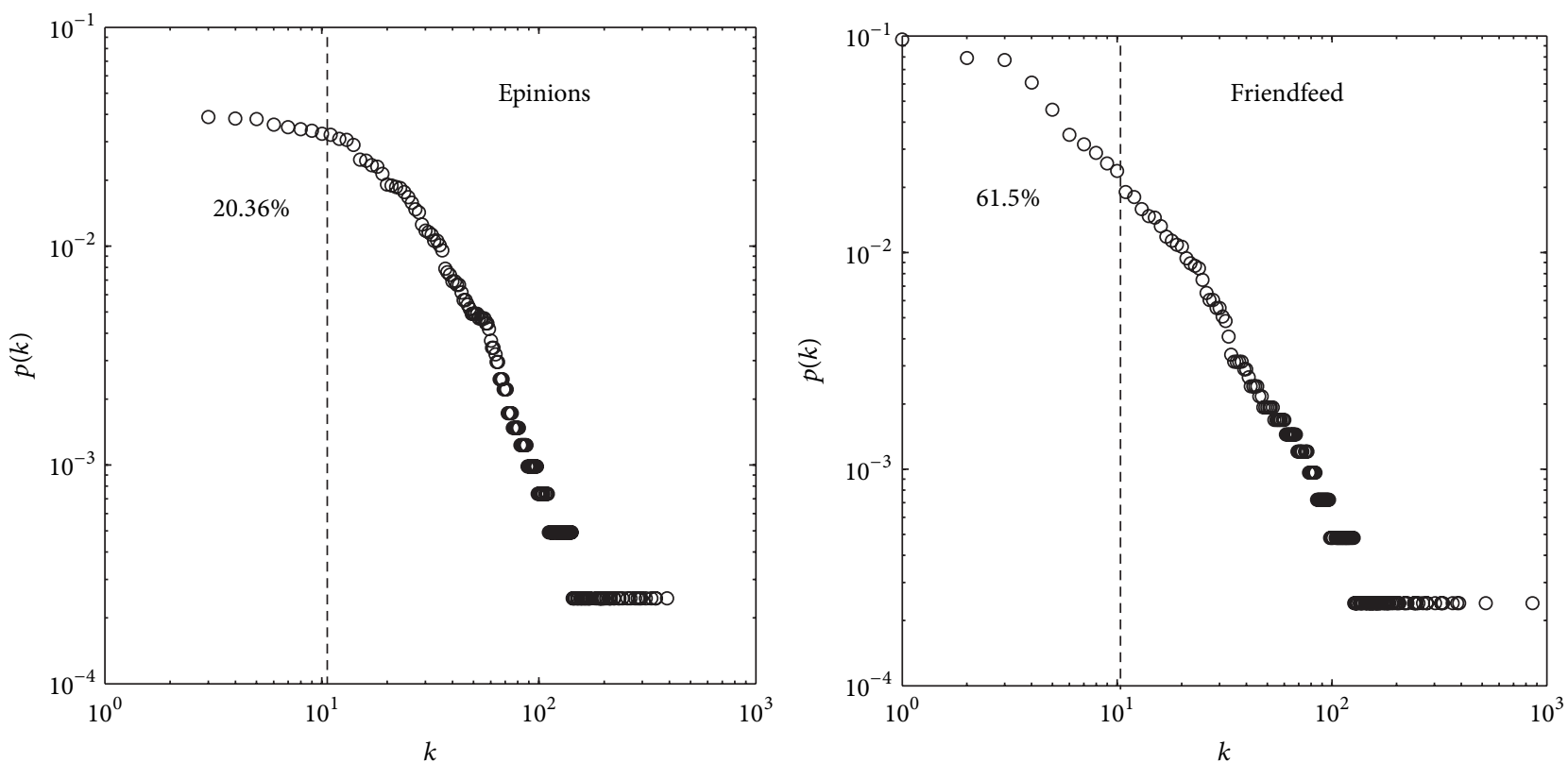

FIGURE 4: The user degree distribution of training set on Epinions and Friendfeed data sets.



(a) Epinions



(b) Friendfeed

Figure 5: Ranking score values venus degree $k$ on Epinions and Friendfeed data sets (color online). The red line, blue line, and green line indicate the performance of BRW, MD, and UCF, respectively. The inset figure amplifies that ranking score versus the degree of users from 1 to 15 .

\section{Acknowledgments}

The authors acknowledge Jun-Lin Zhou for helpful discussions. This work was partially supported by the Natural Science Foundation of China (Grant nos. 61103109, 11105024, and 61300018) and the Special Project of Sichuan Youth Science and Technology Innovation Research Team (Grant no. 2013TD0006).

\section{References}

[1] A. Edmunds and A. Morris, "Problem of information overload in business organizations: a review of the literature," International Journal of Information Management, vol. 20, no. 1, pp. 1728, 2000.

[2] L. Lü, M. Medo, C. H. Yeung, Y. Zhang, Z. Zhang, and T. Zhou, "Recommender systems," Physics Reports, vol. 519, no. 1, pp. 149, 2012. 
[3] L. C. Freeman, "Centrality in social networks conceptual clarification," Social Networks, vol. 1, no. 3, pp. 215-239, 1978.

[4] Y. Ye, J. Yin, and Y. Xu, "Social network supported process recommender system," The Scientific World Journal, vol. 2014, Article ID 349065, 8 pages, 2014.

[5] F. Fu, L. Liu, and L. Wang, "Empirical analysis of online social networks in the age of Web 2.0," Physica A: Statistical Mechanics and Its Applications, vol. 387, no. 2-3, pp. 675-684, 2008.

[6] M. A. Nowak, "Five rules for the evolution of cooperation," Science, vol. 314, no. 5805, pp. 1560-1563, 2006.

[7] G. Szabó and G. Szabó, "Evolutionary games on graphs," Physics Reports, vol. 446, no. 4-6, pp. 97-216, 2007.

[8] S. Fortunato, "Community detection in graphs," Physics Reports A, vol. 486, no. 3-5, pp. 75-174, 2010.

[9] M. N. K. Boulos and S. Wheeler, “The emerging Web 2.0 social software: an enabling suite of sociable technologies in health and health care education," Health Information and Libraries Journal, vol. 24, no. 1, pp. 2-23, 2007.

[10] A. I. Schein, A. Popescul, L. H. Ungar, and D. M. Pennock, "Methods and metrics for cold-start recommendations," in Proceedings of the 25th Annual International ACM SIGIR Conference on Research and Development in Information Retrieval, pp. 253-260, ACM, 2002.

[11] E. Vozalis and K. G. Margaritis, "Analysis of recommender systems algorithms," in Proceedings of the 6th Hellenic European Conference on Computer Mathematics and Its Applications (HERCMA '03), vol. 2003, Athens, Greece, 2003.

[12] F. Radicchi and A. Arenas, "Abrupt transition in the structural format ion of interconnected networks," Nature Physics, vol. 9, pp. 717-720, 2013.

[13] M. de Domenico, A. Sole-Ribalta, E. Cozzo et al., "Mathematical formulation of multilayer networks," Physical Review X, vol. 3 , Article ID 041022, 2013.

[14] S. V. Buldyrev, R. Parshani, G. Paul, H. E. Stanley, and S. Havlin, "Catastrophic cascade of failures in interdependent networks," Nature, vol. 464, no. 7291, pp. 1025-1028, 2010.

[15] M. Givoni and D. Banister, "Airline and railway integration," Transport Policy, vol. 13, no. 5, pp. 386-397, 2006.

[16] T. Zhou, J. Ren, M. Medo, and Y. Zhang, "Bipartite network projection and personal recommendation," Physical Review E: Statistical, Nonlinear, and Soft Matter Physics, vol. 76, no. 4, Article ID 046115, 2007.

[17] T. Zhoua, Z. Kuscsik, J. Liu, M. Medo, J. R. Wakeling, and Y. Zhang, "Solving the apparent diversity-accuracy dilemma of recommender systems," Proceedings of the National Academy of Sciences of the United States of America, vol. 107, no. 10, pp. 45114515, 2010.

[18] P. Resnick, N. Iacovou, M. Suchak, P. Bergstrom, and J. Riedl, "Grouplens: an open architecture for collaborative filtering of netnews," in Proceedings of the ACM Conference on Computer Supported Cooperative Work, pp. 175-186, ACM, 1994.

[19] J. B. Schafer, D. Frankowski, J. Herlocker, and S. Sen, "Collaborative filtering recommender systems," in The adaptive Web, pp. 291-324, Springer, New York, NY, USA, 2007.

[20] G. Adomavicius and A. Tuzhilin, "Toward the next generation of recommender systems: a survey of the state-of-the-art and possible extensions," IEEE Transactions on Knowledge and Data Engineering, vol. 17, no. 6, pp. 734-749, 2005.

[21] J. L. Herlocker, J. A. Konstan, A. Borchers, and J. Riedl, "An algorithmic framework for performing collaborative filtering," in Proceedings of the 22nd Annual International ACM
SIGIR Conference on Research and Development in Information Retrieval, pp. 230-237, 1999.

[22] B. Sarwar, G. Karypis, J. Konstan, and J. Riedl, "Item-based collabo rative filtering recommendation algorithms," in Proceedings of the 10th International Conference on World Wide Web, pp. 285-295, ACM, 2001.

[23] M. Deshpande and G. Karypis, "Item-based top- $N$ recommendation algorithms," ACM Transactions on Information Systems, vol. 22, no. 1, pp. 143-177, 2004.

[24] J. S. Breese, D. Heckerman, and C. Kadie, "Empirical analysis of predicti ve algorithms for collaborative filtering," in Proceedings of the 14th Conference on Uncertainty in Artificial Intelligence (UAI '98), pp. 43-52, Morgan Kaufmann, Madison, Wis, USA, July 1998.

[25] G. Linden, B. Smith, and J. York, "Amazon.com recommendations: item-to-item collaborative filtering," IEEE Internet Computing, vol. 7, no. 1, pp. 76-80, 2003.

[26] M. J. Pazzani and D. Billsus, "Content-based recommendation systems," in The Adaptive Web, pp. 325-341, Springer, 2007.

[27] R. Burke, "Hybrid web recommender systems," in The Adaptive Web, pp. 377-408, Springer, New York, NY, USA, 2007.

[28] C. Palmisano, A. Tuzhilin, and M. Gorgoglione, "Using context to improve predictive modeling of customers in personalization applications," IEEE Transactions on Knowledge and Data Engineering, vol. 20, no. 11, pp. 1535-1549, 2008.

[29] D. C. Nie, M. J. Ding, Y. Fu, J. L. Zhou, and Z. K. Zhang, "Social interest for user selecting items in recommender systems," International Journal of Modern Physics C, vol. 24, no. 4, Article ID 1350022, 2013.

[30] Z. Zhang, T. Zhou, and Y. Zhang, "Tag-aware recommender systems: a state-of-the-art survey," Journal of Computer Science and Technology, vol. 26, no. 5, pp. 767-777, 2011.

[31] K. Pearson, “The problem of the random walk," Nature, vol. 72, no. 1865, p. 294, 1905.

[32] Z. Huang, H. Chen, and D. Zeng, "Applying associative retrieval techniques to alleviate the sparsity problem in collaborative filtering," ACM Transactions on Information Systems, vol. 22, no. 1, pp. 116-142, 2004.

[33] A. Zeng, A. Vidmer, M. Medo, and Y. C. Zhang, "Information filtering by similarity-preferential diffusion processes," Europhysics Letters, vol. 105, Article ID 58002, 2014.

[34] P. Sarkar and A. W. Moore, "Random walks in social networks and their applications: a survey," in Social Network Data Analytics, pp. 43-77, 2011.

[35] A. W. Yu, N. Mamoulis, and H. Su, "Reverse top-k search using random walk with restart," in Proceedings of the VLDB Endowment, vol. 7, 2014.

[36] P. Massa and P. Avesani, "Trust-aware recommender systems," in Proceedings of the ACM Conference on Recommender Systems (RecSys '07), pp. 17-24, ACM, Valley, Calif, USA, October 2007.

[37] I. Esslimani, A. Brun, and A. Boyer, "From social networks to behavioral networks in recommender systems," in Proceedings of the International Conference on Advances in Social Network Analysis and Mining (ASONAM '09), pp. 143-148, IEEE, July 2009.

[38] F. E. Walter, S. Battiston, and F. Schweitzer, "A model of a trust-based recommendation system on a social network," Autonomous Agents and Multi-Agent Systems, vol. 16, no. 1, pp. 57-74, 2008.

[39] C. H. Lai, D. R. Liu, and C. S. Lin, "Novel personal and groupbased trust models in collaborative filtering for document 
recommendation," Information Sciences, vol. 239, pp. 31-49, 2013.

[40] B. Yin, Y. Yang, and W. Liu, "Exploring social activeness and dyna mic interest in community-based recommender system," in Proceedings of the Companion Publication of the 23rd International Conference on World Wide Web Companion, pp. 771-776, International World Wide Web Conferences Steering Committee, 2014.

[41] C. Wei, R. Khoury, and S. Fong, "Web 2.0 Recommendation service by multi-collaborative filtering trust network algorithm," Information Systems Frontiers, vol. 15, no. 4, pp. 533-551, 2013.

[42] D. Crandall, D. Cosley, D. Huttenlocher, J. Kleinberg, and S. Suri, "Feedback effects between similarity and social influence in online communities," in Proceedings of the 14th ACM SIGKDD International Conference on Knowledge Discovery and Data Mining (KDD '08), pp. 160-168, August 2008.

[43] J. L. Herlocker, J. A. Konstan, L. G. Terveen, and J. T. Riedl, "Evaluating collaborative filtering recommender systems," ACM Transactions on Information Systems, vol. 22, no. 1, pp. 553, 2004.

[44] T. Zhou, L. L. Jiang, R. Q. Su, and Y. C. Zhang, "Effect of initial configuration on network-based recommendation," Europhysics Letters, vol. 81, no. 5, Article ID 58004, 2008.

[45] T. Zhou, R. Q. Su, R. R. Liu, L. L. Jiang, B. H. Wang, and Y. Zhang, "Accurate and diverse recommendations via eliminating redundant correlations," New Journal of Physics, vol. 11, Article ID 123008, 2009. 

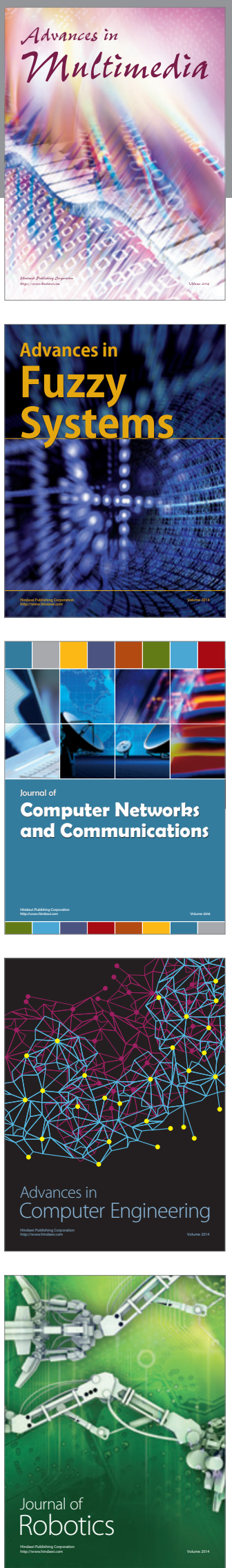

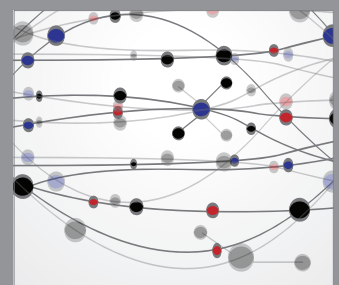

The Scientific World Journal
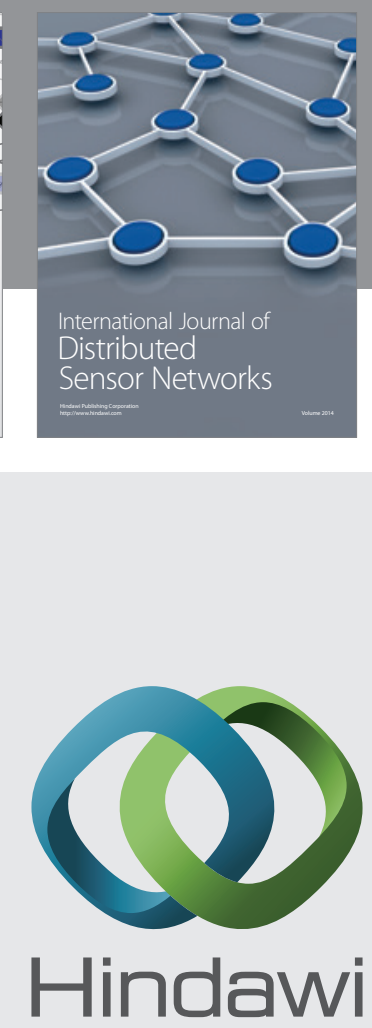

Submit your manuscripts at

http://www.hindawi.com
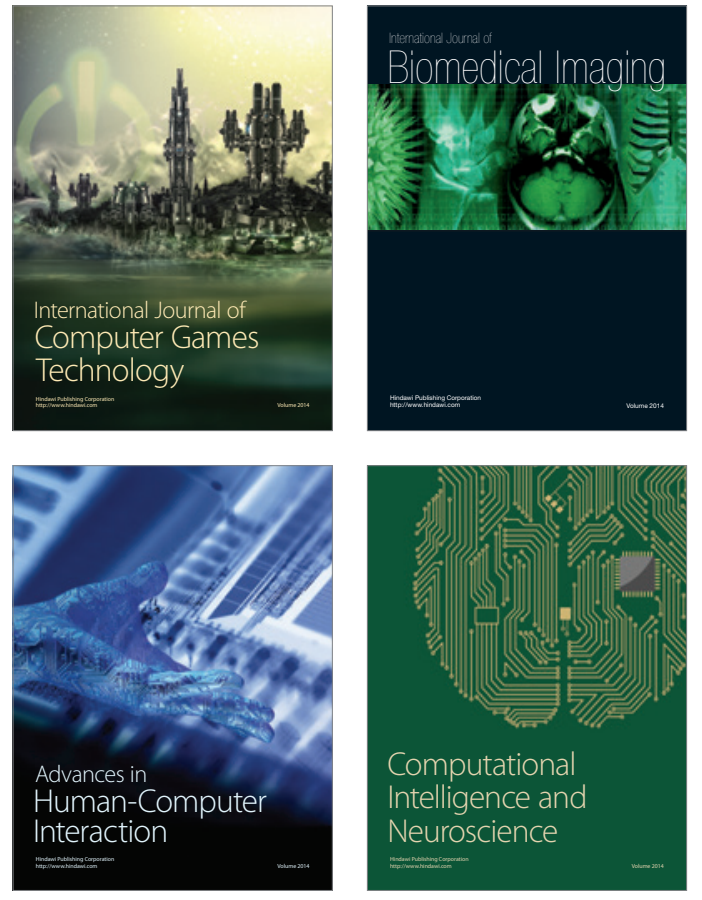
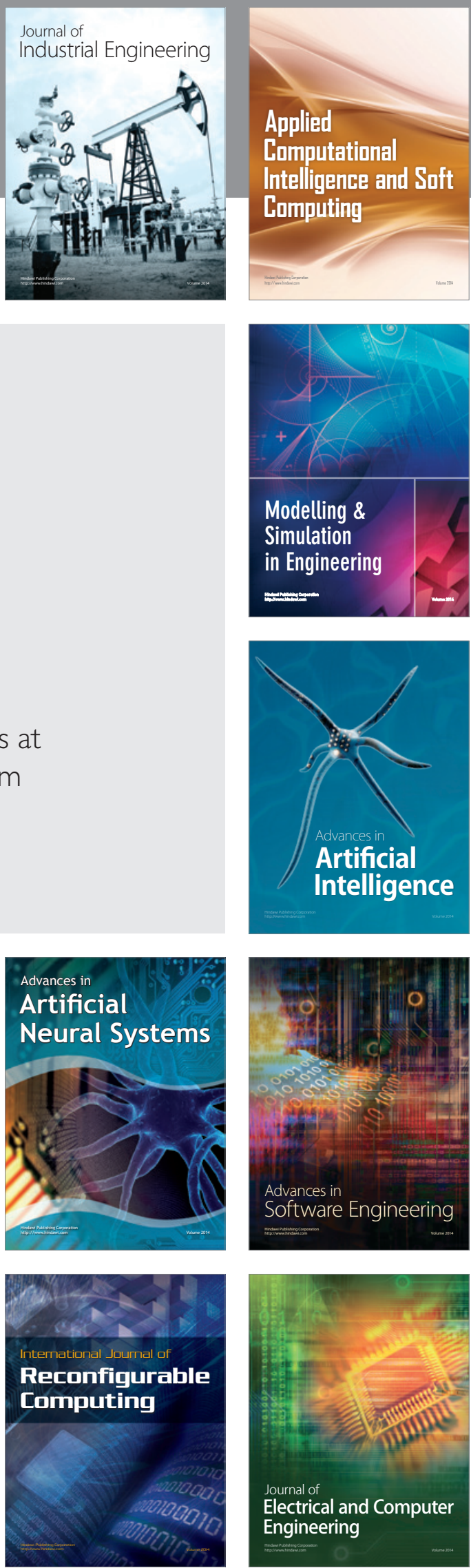